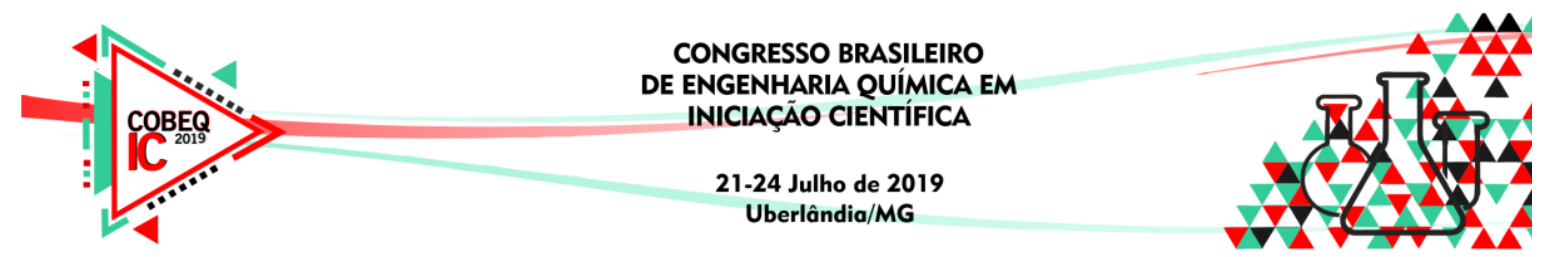

\title{
IMPLEMENTAÇÃO DE UM ALGORITMO HÍBRIDO PARA OTIMIZAÇÃO DE FUNÇÕES BIDIMENSIONAIS
}

\author{
F. S. FREITAS ${ }^{1}$, G. L. STRÖHER ${ }^{2}$ e G. R. STRÖHER ${ }^{1}$ \\ 1, 2, 3 Universidade Tecnológica Federal do Paraná, Departamento de Engenharia Química \\ E-mail para contato: fernandofreitas@alunos.utfpr.edu.br
}

RESUMO - O presente trabalho tem como objetivo implementar um algoritmo híbrido para otimização de funções bidimensionais a partir do sequenciamento de dois tipos de metodologias, não determinísticas e subsequentemente determinística. Na primeira fase o algoritmo proposto utiliza Algoritmo Genético (AG) e na segunda fase o algoritmo Simplex de Nelder - Mead. O Algoritmo Genético provê uma área promissora em que se encontra o mínimo global e na sequência o Simplex é iniciado a fim de identificar o ponto exato, ou o mais preciso possível, em que o mínimo global se encontra. O algoritmo híbrido proposto foi extensivamente testado usando funções Benchmark. Os testes foram realizados variando diversos parâmetros do AG com o objetivo de identificar qual combinação de parâmetros provê os melhores resultados de otimização global em um menor tempo de processamento.

\section{INTRODUÇÃO}

Os Algoritmos Genéticos (AG's) são uma classe de algoritmos que utilizam técnicas inspiradas na biologia evolutiva como hereditariedade, mutação, seleção e recombinação. AG's se basearem em uma população de possíveis soluções e além disso, podem ser implementados sem a necessidade de conhecimento prévio do problema. Necessita-se apenas de uma forma adequada de representação e avaliação das possíveis soluções do problema a ser tratado (LIMA, 2008).

A lenta convergência de AGs antes de fornecer uma solução precisa é um inconveniente bem conhecido. Esta limitação, muitas vezes impede que os AGs sejam realmente de interesse prático para muitas aplicações. Para contornar tal limitação, vários métodos híbridos têm sido propostos na literatura, combinando um AG com algum algoritmo clássico de hill-climbing, basicamente o papel dedicado ao AG ainda é localizar alguma região promissora dentro do amplo espaço de solução, enquanto o algoritmo de otimização clássico permite alcançar, com precisão e rapidez, a melhor solução situada naquela região (CHELOUAH, 2003).

Segundo Chelouah (2003) a maioria dos algoritmos de otimização local existentes podem ser escolhida para cooperar dessa forma com AG. No presente trabalho propõem-se um método híbrido combinando o AG com o Algoritmo Simplex de Nelder-Mead. O algoritmo híbrido proposto visa determinar o mínimo global de funções bidimensionais multimodais na qual o AG tem a função de encontrar uma área promissora e o Simplex na sequência visa a determinar do ponto exato, ou o mais próximo possível em que o mínimo se encontra. 


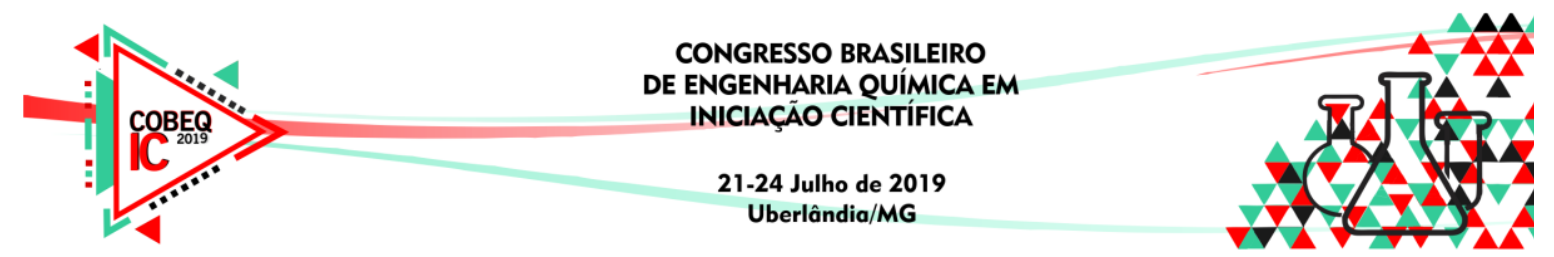

\section{METODOLOGIA}

\subsection{Descrição do Método Híbrido de Otimização}

O algoritmo necessita de uma população inicial de indivíduos na qual contém coordenadas reais associadas a função objetivo. Essa população inicial passa por operadores inspirados na genética de reprodução que simulam acontecimentos biológicos reais aos seres vivos. Descendentes são gerados a partir dos pais, criando assim uma nova população promissora, que é escolhida na seleção dos melhores indivíduos. O mecanismo de reprodução usado foi o crossover, que consiste em reordenar coordenadas de dois indivíduos e mutação que substitui uma coordenada de um indivíduo. Após o AG definir uma área promissora onde se encontra o mínimo global, o Simplex é iniciado afim de identificar o ponto mínimo no qual ele não encontraria sem o auxílio do AG.

As principais características dos métodos utilizados no AG, assim como alguns parâmetros foram os seguintes: geração da população inicial, operadores de seleção que basicamente seleciona os indivíduos com melhores avaliações, operadores de recombinação (crossover) que gera descendentes a partir dos melhores indivíduos escolhidos, operador de mutação que agrega diversidade a população e elitismo onde garante que os melhores indivíduos de cada geração não morram.

O algoritmo Simplex pertence a uma classe mais geral de algoritmos de pesquisa direta e que não faz uso de derivadas, portanto trata-se de uma técnica de otimização não linear, baseada no método de otimização livre de derivada, Derivative-Free Optimization (DFO). O Simplex busca, a partir de uma primeira solução básica viável, percorrer de forma iterativa os vértices de um polígono até alcançar uma solução considerada ótima para o problema. Constitui-se de um método que minimiza uma função de $\mathrm{M}$ variáveis que, por sua vez, depende da comparação da função nos seus $(M+1)$ vértices de um simplex geral seguido pela recolocação do vértice com o valor mais alto por outro ponto de menor valor na função objetivo. De um ponto de vista computacional, esse método mostra-se ser compacto e efetivo (GOMES, 2017).

\subsection{Critério de Parada}

O algoritmo gera uma população inicial de indivíduos que contemple todo o domínio de busca estipulado inicialmente, na sequência é calculado os respectivos valores da função objetivo para cada indivíduo. A nova população é gerada a partir dessa por meio das etapas genéticas que já foram descritas: seleção, recombinação e mutação. A nova população criada a partir da antiga passa para a próxima geração onde o processo é reiterado. O processo iterativo só é interrompido quando atingir o número máximo de gerações definido inicialmente ou uma precisão relacionada as coordenadas dos indivíduos, $\rho_{a b s}$, dada pela Equação 1 .

$$
\frac{1}{N^{\circ}{ }_{\text {ind }}} \sum_{i}^{N^{\circ}{ }^{i n d}} \| \operatorname{Ind}_{i}-\text { MelhorInd } \| \leq \rho_{a b s}
$$




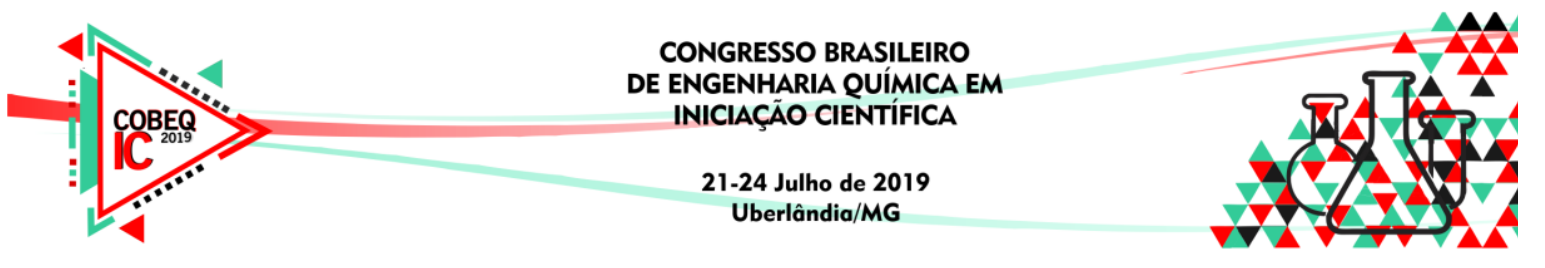

Para exemplificar, é calculado a distância entre um os indivíduos da geração atual, ind, com o melhor ponto encontrado até o momento, MelhorInd, sendo que, a iteração é interrompida caso esse valor dê menor que $\rho_{a b s}$, sendo também definido inicialmente.

Assim que um dos critérios de parada do AG é atingido o algoritmo Simplex é então acionado. O Simplex recebe a coordenada do ponto mínimo a partir do melhor indivíduo gerado pelo AG, e esse ponto mínimo passa a ser solução inicial, ou seja, um dos vértices da forma geométrica, em geral ortogonal, do Simplex.

A iteração realizada pelo Simplex, já descrita acima, só é interrompida quando pelo menos um dos dois critérios de parada são atingidos. O primeiro critério de parada é satisfeito quando o algoritmo atingir o número de iterações máximas estipulado inicialmente, $k$, o que em geral acaba não acontecendo. $\mathrm{O}$ outro critério de parada, normalmente o que é satisfeito primeiro, está relacionado ao cálculo do desvio padrão dos vértices $n+1$ de um Simplex avaliados na função objetivo. As interações são interrompidas se o cálculo do desvio padrão, Equação 2, for menor que um erro pré-definido, Er. O próprio simplex adapta-se à paisagem local e contrai-se a um mínimo local final.

$$
\sqrt{\frac{\sum_{i=1}^{n+1}\left(f_{i}-\bar{f}\right)^{2}}{n+1}} \leq E r
$$

Em que $f_{i}$ é o valor dos $n+1$ vértices de um Simplex avaliados na função objetivo, $n$ é o número de variáveis da função objetivo e $\bar{f}$ é a média aritmética dos vértices.

\section{RESULTADOS E DISCUSSÕES}

A fim de determinar a eficiência do algoritmo híbrido, várias funções bidimensionais Benchmark foram testadas. Uma vez que o algoritmo genético não é método determinístico, cada vez que o programa for executado um novo histórico de gerações pode ser obtido, assim seguindo a recomendação de CHELOUAH e SIARRY (2003), cada função foi testada cem vezes. Os testes foram realizados variando diversos operadores do AG com o objetivo de identificar qual combinação de parâmetros provê os melhores resultados de otimização global em um menor tempo de processamento.

Foram utilizadas seis funções Benchmark variando os seguintes operadores genéticos: crossover, mutação e método de seleção. Para avaliar a eficiência do Algoritmo Híbrido, AH, foram estipulados os seguintes critérios obtidos a partir de 100 minimizações por função: taxa de minimizações bem-sucedidas (T.M.B.), média do número de avaliações da função objetivo (M.FOBJ), média entre o melhor ponto encontrado com o ótimo global conhecido (M.GL) e o tempo médio de processamento (T.M.P).

Quando os critérios de paradas do AG e na sequência do Simplex são atingidos o AH fornece as coordenadas de um ponto e o valor da função objetivo avaliado nesse ponto FOBJAH. O resultado é então comparado com o mínimo analítico conhecido da função FOBJANAL, para o julgamento do resultado obtido ser dado como bem-sucedido optou-se em utilizar o requisito apresentado na literatura, mencionada anteriormente, dada pela Equação 3. 


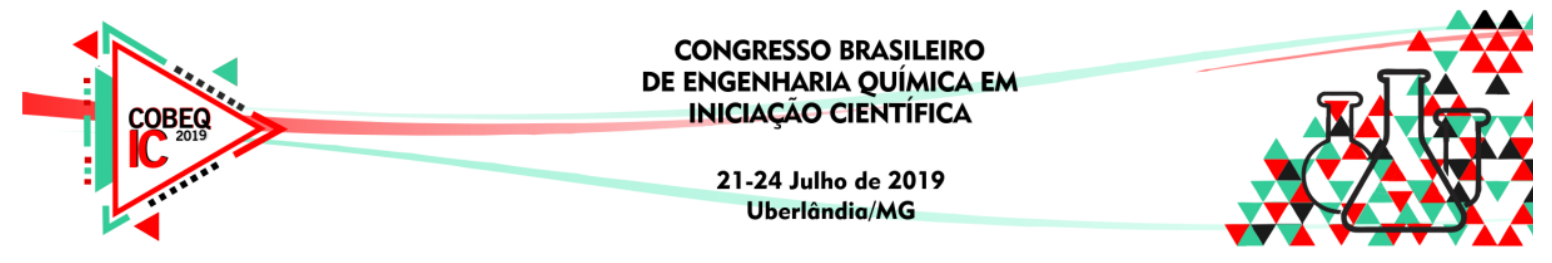

$$
\left|F O B J_{A H}-F O B J_{A N A L}\right|<\varepsilon_{r e l}\left|F O B J_{I N I T}\right|+\varepsilon_{a b s}
$$

em que $\varepsilon_{r e l}=10^{-4}, \varepsilon_{a b s}=10^{-6}$ e FOBJ $J_{I N I T}$ é a média experimental do valor da função objetivo calculada em cem pontos selecionados aleatoriamente antes que o algoritmo seja executado.

Os resultados obtidos variando os operadores genéticos já mencionados são apresentados nas Tabelas 1 a 8 . Vale ressaltar que variações do tempo de processamento podem ocorrer pois o mesmo depende fortemente da capacidade de processamento do computador utilizado. As condições para o tempo de processamento que serão apresentados estão sujeitas a um microcomputador com a configuração Windows 7 Ultimate, processador Intel $®$ CoreTM i32310M CPU @ 2.10 GHz, memória (RAM) de 4 GB, sistema operacional de 64 Bits.

Tabela 1 - Torneio/ Dois pontos/ Mutação de $2,5 \%$.

\begin{tabular}{ccccc}
\hline $\begin{array}{c}\text { Função } \\
\text { teste }\end{array}$ & $\begin{array}{c}\text { T.M.B. } \\
\text { (\%) }\end{array}$ & M.OBJ. & M.GL. & $\begin{array}{c}\text { T.M.P. } \\
\text { (s) }\end{array}$ \\
\hline RC & 100 & 37 & $3.7191 \mathrm{e}-07$ & 0.09 \\
ES & 90 & 388 & $9.6047 \mathrm{e}-09$ & 4.02 \\
GP & 100 & 75 & $9.4281 \mathrm{e}-09$ & 0.31 \\
B2 & 98 & 422 & $8.9496 \mathrm{e}-09$ & 5.95 \\
R2 & 100 & 66 & $1.1844 \mathrm{e}-08$ & 0.13 \\
Z2 & 100 & 38 & $1.4429 \mathrm{e}-08$ & 0.14 \\
\hline
\end{tabular}

Tabela 3 - Amostragem Estocástica Uniforme/ Dois pontos/ Mutação de 2,5\%.

\begin{tabular}{ccccc}
\hline $\begin{array}{c}\text { Função } \\
\text { teste }\end{array}$ & $\begin{array}{c}\text { TMB } \\
(\boldsymbol{\%})\end{array}$ & M.OBJ & M.GL & $\begin{array}{c}\text { T.M.P } \\
\text { (s) }\end{array}$ \\
\hline RC & 100 & 40 & $3.7160 \mathrm{e}-07$ & 0.004 \\
ES & 96 & 463 & $1.1670 \mathrm{e}-08$ & 3.50 \\
GP & 98 & 62 & $1.1134 \mathrm{e}-08$ & 0.22 \\
B2 & 81 & 492 & $1.1537 \mathrm{e}-08$ & 3.77 \\
R2 & 100 & 94 & $1.5956 \mathrm{e}-08$ & 0.48 \\
Z2 & 100 & 267 & $1.2879 \mathrm{e}-08$ & 2.00 \\
\hline
\end{tabular}

Tabela 5 - Torneio/ Uniforme/ Mutação de 2,5\%.

\begin{tabular}{ccccc}
\hline $\begin{array}{c}\text { Função } \\
\text { teste }\end{array}$ & $\begin{array}{c}\text { TMB } \\
(\%)\end{array}$ & M.OBJ & M.GL & $\begin{array}{c}\text { T.M.P } \\
\text { (s) }\end{array}$ \\
\hline RC & 98 & 40 & $3.6806 \mathrm{e}-07$ & 0.1 \\
ES & 91 & 425 & $1.0615 \mathrm{e}-08$ & 5.01 \\
GP & 100 & 80 & $9.7534 \mathrm{e}-09$ & 0.8 \\
B2 & 93 & 436 & $1.0269 \mathrm{e}-08$ & 6.64 \\
R2 & 100 & 68 & $1.0423 \mathrm{e}-08$ & 0.08 \\
Z2 & 100 & 38 & $1.1902 \mathrm{e}-08$ & 0.066 \\
\hline
\end{tabular}

Tabela 2 - Roleta/ Dois pontos/ Mutação de $2,5 \%$.

\begin{tabular}{ccccc}
\hline $\begin{array}{c}\text { Função } \\
\text { teste }\end{array}$ & $\begin{array}{c}\text { TMB } \\
\text { (\%) }\end{array}$ & M.OBJ & M.GL & $\begin{array}{c}\text { T.M.P } \\
\text { (s) }\end{array}$ \\
\hline RC & 100 & 42 & $3.7199 \mathrm{e}-07$ & 0.004 \\
ES & 96 & 496 & $1.5547 \mathrm{e}-08$ & 4.93 \\
GP & 96 & 53 & $1.5037 \mathrm{e}-08$ & 0.19 \\
B2 & 92 & 498 & $1.2512 \mathrm{e}-08$ & 7.23 \\
R2 & 100 & 176 & $1.5113 \mathrm{e}-08$ & 2.35 \\
Z2 & 100 & 317 & $8.8484 \mathrm{e}-09$ & 4.16 \\
\hline
\end{tabular}

Tabela 4 - Torneio/ Um ponto/ Mutação de $2,5 \%$.

\begin{tabular}{ccccc}
\hline $\begin{array}{c}\text { Função } \\
\text { teste }\end{array}$ & $\begin{array}{c}\text { TMB } \\
\text { (\%) }\end{array}$ & M.OBJ & M.GL & $\begin{array}{c}\text { T.M.P } \\
\text { (s) }\end{array}$ \\
\hline RC & 100 & 39 & $3.7127 \mathrm{e}-07$ & 0.085 \\
ES & 95 & 383 & $1.4582 \mathrm{e}-08$ & 3.43 \\
GP & 99 & 70 & $9.4616 \mathrm{e}-09$ & 0.27 \\
B2 & 98 & 444 & $1.2547 \mathrm{e}-08$ & 6.57 \\
R2 & 99 & 64 & $1.1700 \mathrm{e}-08$ & 0.19 \\
Z2 & 100 & 37 & $1.2717 \mathrm{e}-08$ & 0.17 \\
\hline
\end{tabular}

Tabela 6 - Torneio/ baseado em Maioria/ Mutação de 2,5\%.

\begin{tabular}{ccccc}
\hline $\begin{array}{c}\text { Função } \\
\text { teste }\end{array}$ & $\begin{array}{c}\text { TMB } \\
(\%)\end{array}$ & M.OBJ & M.GL & $\begin{array}{c}\text { T.M.P } \\
\text { (s) }\end{array}$ \\
\hline RC & 99 & 40 & $3.6986 \mathrm{e}-07$ & 0.11 \\
ES & 92 & 470 & $1.2615 \mathrm{e}-08$ & 9.89 \\
GP & 94 & 46 & $1.2941 \mathrm{e}-08$ & 0.12 \\
B2 & 92 & 423 & $1.0828 \mathrm{e}-08$ & 7.97 \\
R2 & 100 & 68 & $1.3403 \mathrm{e}-08$ & 0.12 \\
Z2 & 100 & 38 & $1.0105 \mathrm{e}-08$ & 0.12 \\
\hline
\end{tabular}




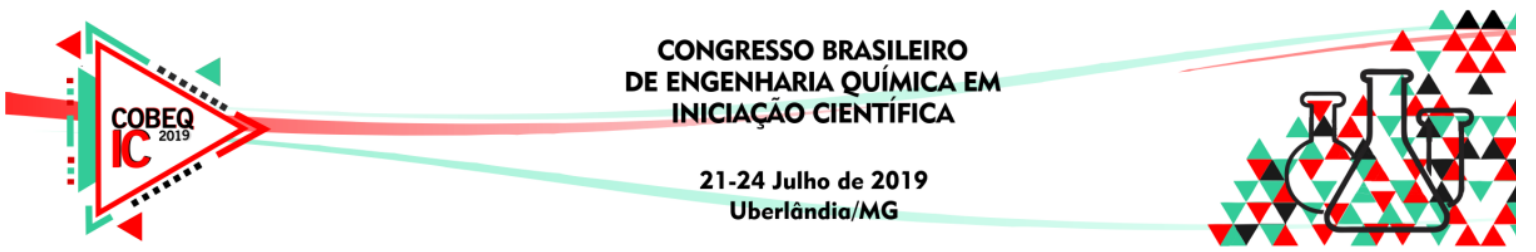

Tabela 7 - Torneio/ Dois pontos/ Mutação de $5 \%$.

\begin{tabular}{ccccc}
\hline $\begin{array}{c}\text { Função } \\
\text { teste }\end{array}$ & $\begin{array}{c}\text { TMB } \\
(\%)\end{array}$ & M.OBJ & M.GL & $\begin{array}{c}\text { T.M.P } \\
\text { (s) }\end{array}$ \\
\hline RC & 100 & 40 & $3.6991 \mathrm{e}-07$ & 0.11 \\
ES & 78 & 208 & $1.3576 \mathrm{e}-08$ & 2.3 \\
GP & 97 & 49 & $1.1849 \mathrm{e}-08$ & 0.18 \\
B2 & 80 & 267 & $1.2256 \mathrm{e}-08$ & 2.5 \\
R2 & 100 & 68 & $1.3053 \mathrm{e}-08$ & 0.16 \\
Z2 & 100 & 37 & $1.0655 \mathrm{e}-08$ & 0.06 \\
\hline
\end{tabular}

Tabela 8 - Torneio/ Dois pontos/ Mutação de $0 \%$.

\begin{tabular}{ccccc}
\hline $\begin{array}{c}\text { Função } \\
\text { teste }\end{array}$ & $\begin{array}{c}\text { TMB } \\
(\boldsymbol{\%})\end{array}$ & M.OBJ & M.GL & $\begin{array}{c}\text { T.M.P } \\
\text { (s) }\end{array}$ \\
\hline RC & 100 & 40 & $3.7185 \mathrm{e}-07$ & 0.09 \\
ES & 70 & 126 & $1.0347 \mathrm{e}-08$ & 1.8 \\
GP & 88 & 127 & $1.0759 \mathrm{e}-08$ & 0.1 \\
B2 & 76 & 54 & $1.4743 \mathrm{e}-08$ & 0.33 \\
R2 & 100 & 68 & $1.7287 \mathrm{e}-08$ & 0.1 \\
Z2 & 100 & 44 & $1.1922 \mathrm{e}-08$ & 0.24 \\
\hline
\end{tabular}

As Tabelas 1 a 3 apresentam resultados obtidos variando-se o método de seleção onde observa-se que os valores obtidos entre elas tiveram pequenas variações, todavia, ao analisar algumas funções específicas, como por exemplo a função B2 observa-se uma pequena variação nos valores da T.M.B. em que o melhor resultado esteve presente no método de seleção torneio apresentado na Tabela 1. Outro exemplo é a função Z2 que apresentou uma M.OBJ. muito menor no torneio, promovendo assim um menor tempo de processamento, uma vez que o número de avaliações da função objetivo foi menor, e por fim, o M.G.L. para o torneio, ao contrário dos outros dois métodos, esteve na faixa de $10^{-9}$ para a maioria das funções avaliadas o que reflete diretamente na precisão dos resultados obtidos.

Fixando-se o torneio, pelos motivos já comentados, para o método de seleção dos indivíduos, avaliou-se, na sequência, os operadores genéticos de crossover, um ponto, dois pontos, uniforme e baseado em maioria cujos os resultados são sumarizados nas Tabelas 1, 4, 5 e 6 , respectivamente. Os resultados obtidos pelos diferentes métodos sinalizam que os operadores de crossover avaliados não influenciaram significativamente o desempenho do A.H., sendo que a influência mais aparente foi com relação as T.M.B. que teve maiores taxas para o operador de dois pontos apresentado na Tabela 1. A T.M.P., associado ao erro do valor da função objetivo, foi menor para o operador de dois pontos também, estando na ordem de $10^{-}$ ${ }^{9}$ para a maioria das funções analisadas.

Por fim, variou-se a probabilidade de mutação, que é fundamental para todo AG pois é ela que garante a continuidade da existência de diversidade genética na população. Os testes realizados até então foram com 2,5\% de mutação, e nas Tabelas 7 e 8 , utilizou-se $5 \%$ e $0 \%$ de mutação respectivamente. Como já era esperado, quando não houve mutação os resultados de T.M.B. foram os piores já obtidos, isso se deve ao fato de que, segundo Linden (2012), quando a probabilidade de mutação for baixo demais, o AG agirá de forma extremamente parcimoniosa e a população não terá diversidade depois de um certo número de gerações, estagnando bem rápido devido à convergência genética.

Enquanto que para a probabilidade de mutação de $5 \%$, os resultados também não foram tão satisfatórios quanto para a mutação de $2,5 \%$, sendo a melhor para todas as funções analisadas. Uma taxa probabilidade de mutação muito alta o AG apresenta um comportamento similar a um algoritmo aleatório (random walk) e perderá suas características interessantes. 


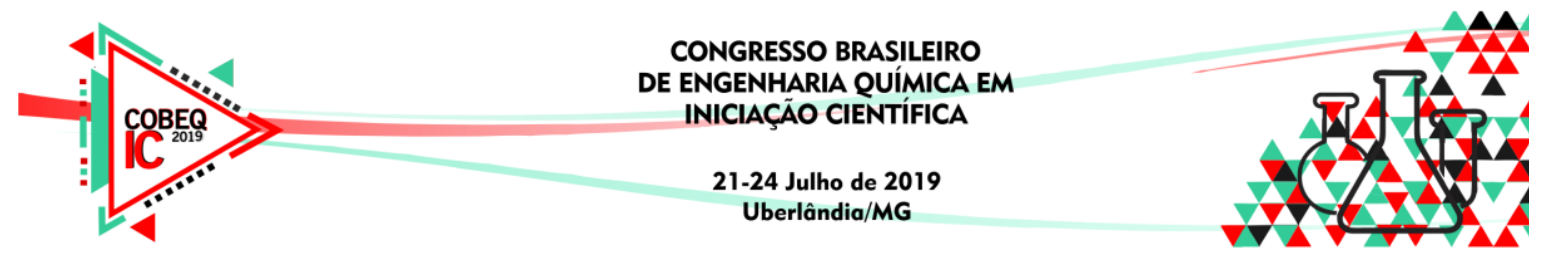

\section{CONCLUSÃO}

No presente trabalho desenvolveu-se um algoritmo híbrido, $\mathrm{AH}$, para otimização global de funções bidimensionais em que o mesmo foi testado com algumas funções Benchmark. As várias combinações de parâmetros do algoritmo genético foram testadas e a combinação que apresentou os melhores resultados foi o método de seleção torneio e o crossover de dois pontos com uma probabilidade de mutação de 2,5\%. Após a identificação da área promissora pelo AG, o Simplex de Nelder - Mead apresentou rápida convergência na determinação do ponto de mínimo global.

\section{REFERÊNCIAS}

CHELOUAH, Rachid; SIARRY, Patrick. Genetic and Nelder-Mead algorithms hybridized for a more accurate global optimization of continuous multiminima functions. European Journal of Operational Research, v. 148, n. 2, p. 335-348, 2003.

GOMES, F. I. F. N. et al. Estratégia híbrida de otimização sem derivadas para a inversão completa da forma da onda. Holos, v. 33, n. 2, p. 2-34, 2017.

LIMA, Ednaldo Oliveira. Algoritmo genético híbrido aplicado à otimização de funções. Trabalho de conclusão de curso, UFES, 2008.

LINDEN, Ricardo. Algoritmos genéticos. 3 Ed. Rio de Janeiro: Editora Ciência Moderna Ltda., 2012. 